\title{
Abnormal myocardial perfusion in the absence of anatomically significant coronary artery disease: Implications and clinical significance
}

\author{
Shreenidhi M. Venuraju, MBBS, MRCP, ${ }^{\text {a }}$ Ajay Yerramasu, MBBS, MRCP, \\ and Avijit Lahiri, MBBS, MSc, MRCP, FACC, FESC ${ }^{\text {a,b,c }}$
}

\section{See related article, pp. 38-44}

\section{DIAGNOSTIC AND PROGNOSTIC VALUE OF MYOCARDIAL PERFUSION SCINTIGRAPHY}

Myocardial Perfusion Scintigraphy (MPS) has dominated the field of noninvasive cardiac imaging for over three decades now and continues to do so largely due to the unparalleled evidence supporting its diagnostic and prognostic value in the management of coronary artery disease (CAD). In recent years, there has been a dramatic rise in the number of cardiac imaging procedures that are being undertaken. In the USA alone, over 10 million myocardial perfusion scans are being performed annually. ${ }^{1}$ This is driven by a need to be able to better risk stratify patients with suspected CAD, particularly those in the intermediate risk group in whom the clinical management decisions are often unclear. From a clinician's standpoint, the value of MPS lies in its ability to clearly guide decisions regarding coronary revascularization ${ }^{2}$; hence the clinical interest in this technique remains unabated over the years.

Two of the most important factors affecting prognosis of patients with CAD are: the extent of myocardium at risk and left ventricular function. MPS by gated Single Photon Emission Computed Tomography (SPECT) is uniquely placed to provide important prognostic data on

From the Clinical Imaging and Research Centre, ${ }^{\mathrm{a}}$ The Wellington Hospital, London, United Kingdom; University of Middlesex ${ }^{\mathrm{b}}$ and Imperial College, ${ }^{\mathrm{c}}$ London, United Kingdom.

Reprint requests: Shreenidhi M. Venuraju, MBBS, MRCP, Clinical Imaging and Research Centre, The Wellington Hospital, Wellington Place, London NW8 9LE, United Kingdom; shreenidhimv@gmail. com.

J Nucl Cardiol 2010;17:8-12.

$1071-3581 / \$ 34.00$

Copyright () 2009 by the American Society of Nuclear Cardiology.

doi:10.1007/s12350-009-9143-4 both these parameters. In patients with suspected CAD, MPS acts as an useful gatekeeper to invasive coronary angiography and has been shown to be cost-effective for this purpose. ${ }^{3}$ MPS also yields independent and incremental prognostic information to that obtained from clinical and stress ECG data. ${ }^{4}$ The prognostic value of the extent of myocardium at risk (as assessed by extent of reversible perfusion defects with Tl-201), and its subsequent relationship with cardiac death was initially described in a study of 100 patients with no known history of myocardial infarction who presented for evaluation of chest pain. ${ }^{5}$ Since then, there have been a multitude of studies documenting the ability of abnormal MPS in predicting future cardiac deaths, nonfatal myocardial infarction and revascularization. ${ }^{6,7}$ Equally, a negative/ normal MPS was shown to be associated with a good prognostic outcome. ${ }^{8,9}$ Of note, majority of the data regarding the diagnostic and prognostic utility of MPS comes from studies that included patients with significant CAD (either $>50 \%$ or $>70 \%$ luminal stenosis in major epicardial vessels).

\section{PERFUSION ABNORMALITIES IN THE ABSENCE OF ANATOMICALLY SIGNIFICANT DISEASE: IS IT PROGNOSTICALLY RELEVANT?}

In this journal, Adamu and colleagues ${ }^{10}$ have reported the results of a 6.3-year follow-up of 123 patients who have had a stress myocardial SPECT with Tc-99m sestamibi or Tetrofosmin followed by invasive coronary angiography within 3 months. All the patients included in the study had proven nonsignificant coronary disease $(<50 \%$ reduction in diameter by quantitative coronary angiography). The end points for this study were death, a composite of death, nonfatal myocardial infarction, and revascularization by coronary artery bypass surgery or per-cutaneous coronary intervention (PCI) and the occurrence of symptoms such as chest pain (CCS class II to IV) and/or breathlessness (NYHA class II to IV). Adamu and colleagues ${ }^{10}$ were rigorous in their inclusion criteria and excluded patients with confounding factors such as cardiomyopathy, left bundle branch block, valvular disease, and history of myocardial infarction. 
During the follow-up period, a total of 17 deaths and 10 myocardial infarctions were noted, giving an annual mortality rate of $2.1 \%$. In a multivariate model that included age, gender, diabetes, stress level, sum rest score (SRS), sum stress score (SSS) and sum difference score (SDS), SSS and age were the only predictors of mortality, while both SSS and Diabetes predicted major adverse cardiac events (MACE). The figures of annualised mortality rates for patients with SSS $<13$ at $1.3 \%$ and for those with SSS $>13$ at $4.5 \%$ are significant indeed.

Interesting as these findings are, they have to be interpreted with caution, in light of some limitations of this study. Perhaps the most important limitation is the relatively small number of patients, both the total number of patients as well those with significant perfusion abnormality. Out of the possible 1667 patients who underwent coronary angiography and MPS within 3 months, only 123 patients were included in the study, reflecting the rigorous patient selection criteria. More importantly, MPS was either normal or only mildly abnormal in the vast majority of these patients (SSS $>4$ in 50 patients), with only 16 patients showing severe perfusion abnormality (defined as SSS $>14)$ and even fewer patients $(n=6)$ showing significant reversible perfusion abnormality (SDS $>8$ ). This is, however, not surprising given that abnormal perfusion is rarely encountered clinically in the absence of significant coronary stenosis.

Although all patients included in this analysis underwent coronary angiography and quantitative coronary angiography (QCA) when a lesion was identified visually, the authors have not included this data in the results. We consider this a significant omission for three reasons: (1) Histopathological and intra-vascular ultrasound studies ${ }^{11,12}$ have clearly demonstrated the propensity of angiography to underestimate lesional severity. And therefore it is likely that some of the borderline lesions may have been classified as non-significant though may have been causing more than $50 \%$ stenosis. The three factors identified by IVUS ${ }^{13}$ that have been shown to predict ischemia with greater sensitivity and specificity are lesion percent plaque burden of $\geq 75 \%$, lesion percent stenosis of $>60 \%$, and a residual luminal area of $\leq 4 \mathrm{~mm}^{2}$. (2) It is conceivable that the angiographic lesions (though not significantly stenotic) could account for at least some of the hard coronary events observed. It is well known that most of the acute coronary events result from the sudden rupture and subsequent thrombosis of plaques that are vulnerable but not necessarily stenotic. ${ }^{14}$ The propensity of a plaque to rupture is determined by its composition and the inflammatory activity within it, rather than the degree of luminal narrowing caused by it. ${ }^{15}$ Therefore, patients with plaques will be at a higher of risk of acute coronary events than those with no plaques, and the more plaques a patient has, greater should be this risk. (3) Some of the angiographic lesions, although not significantly obstructive at the time of initial angiography, could have progressed during the course of follow-up and accounted for the symptoms subsequently observed. In this regard, it is pertinent to note that the presence and extent of plaques at baseline are significant determinants of subsequent plaque progression. ${ }^{16}$ (4) It is possible that some of these anatomically "nonsignificant" lesions are flow-limiting. In fact, a similar study by Brown et $\mathrm{al}^{17}$ showed that abnormal MPS results were more common in patients with 21-40\% maximal stenosis than in patients with $0-20 \%$ maximal stenosis $(59 \%$ vs $27 \% ; P<0.01)$. Factors other than the degree of luminal stenosis, such as the length of the lesion, presence of serial lesions, residual vessel diameter, vasomotor tone, and blood viscosity (as indicated by the Hagen-Poiseuille law) can influence the dynamics of blood flow across a lesion. For instance, two coronary lesions in tandem, each associated with $40 \%$ stenosis, might cause a greater reduction in blood flow than a single $\geq 50 \%$ stenotic lesion. Hence, the inclusion of angiographic data in the multivariate model could have influenced the prognostic value of MPS, given that some degree of colinearity is possible between these two variables and some of the outcomes could have been explained by the angiographic data.

Other limitations pertain to the technique of MPS itself. The authors did not mention the use of any attenuation correction in the study. Some of the smaller perfusion defects (especially the non-reversible defects in the inferior wall) that have been picked up by the software programme could represent attenuation artefacts, which is a problem often encountered with SPECT. A detailed analysis of the distribution of the perfusion defects is missing, which could have shed further light on the possible impact of attenuation artefacts.

Not withstanding the above limitations, the study is likely to be an important addition to the MPS literature dominated by studies evaluating the prognostic ability of this technique in patients with significant obstructive CAD. The results of this study, if replicated in larger trials, can extend the prognostic value of MPS to the group of patients without anatomically significant CAD.

\section{DISCORDANCE BETWEEN THE ANATOMICAL AND FUNCTIONAL ASPECTS OF CORONARY ARTERY DISEASE}

Adamu et $\mathrm{al}^{10}$ also offer some intriguing insights into the pathophysiology of $\mathrm{CAD}$, in particular, the complex relationship between the anatomical severity and functional significance of coronary atherosclerosis. Although it is a common notion that the severely stenotic plaques are more likely to be associated with perfusion abnormalities, such an association is not necessarily linear and is influenced largely by factors such as the development of 
collateral circulation. In clinical practice, it is not uncommon to encounter patients with severe coronary stenosis $(>70 \%)$ and extensive atherosclerosis (Coronary Calcium Score $>1000 \AA$ units) who show completely normal perfusion on MPS. More importantly, a normal perfusion scan is associated with a good prognostic outcome, even in the presence of extensive coronary atherosclerosis. ${ }^{18}$ The findings of the study by Adamu et al suggest that the converse is also true, i.e., not only is it possible to see severe perfusion defects in patients without anatomically significant coronary lesions, but such defects are also of prognostic importance.

\section{MICROVASCULAR DISEASE-MYTH OR REALITY}

Adamu et al conclude that perfusion defects assessed quantitatively must represent more widespread atherosclerosis than is apparent with the luminal study of the major epicardial vessels, i.e., involving the coronary microvasculature. However, it is difficult to presume that microvascular disease alone was responsible for all of the observed perfusion defects in this cohort. It is difficult to envisage a SSS of $>14$ in the absence of any significant stenosis in the epicardial vessels. Even if we consider that microvascular disease was responsible for some, if not all, of the perfusion abnormalities noted in this study, involvement of the coronary microvasculature is notoriously difficult to diagnose and academic interest in it has waxed and waned in the last three decades. Various techniques have been used to assess coronary microvascular function. Almost all of these techniques are confined to the realms of academia and are not routinely used in clinical practice. Invasive Doppler measurements, Positron Emission Tomography (PET), SPECT, Contrast Echocardiography and more recently cardiac MRI, and multislice cardiac CT have all been used.

Absolute myocardial blood flow in terms of $\mathrm{mL} / \mathrm{min} / \mathrm{g}$ can be measured accurately with PET using O-15, N-13, or Rubidium-82, and is therefore the gold standard for evaluating coronary flow reserve (CFR: ratio of absolute myocardial blood flow at baseline and during maximal hyperemia). Previous studies with PET have demonstrated that CFR is reduced before plaque lesions or luminal stenoses are apparent by either invasive angiography or multislice computed tomography (MSCT). ${ }^{19,20}$ This reduced coronary flow reserve has been attributed to endothelial and/or microvascular dysfunction.

Though quantification of MBF with SPECT has been studied in small cohorts of patients, ${ }^{21-23}$ there is certainly a dearth of data regarding its prognostic value. There have also been a few doubts raised regarding the technique itself. First with Tl-201, washout analysis is carried out as a surrogate marker of CFR, though it is known that endothelial dependent vasodilatation is not the only criteria affecting Tl-201 washout characteristics. ${ }^{24}$ Second, with Sestamibi or Tetrofosmin, there is absence of significant redistribution requiring two injections of the radio-tracer at different time intervals when there may be differences in underlying cardiac physiological characteristics. There is also a nonlinear relationship between myocardial blood flow and tracer uptake, especially at higher flow rates. ${ }^{25}$ Despite these setbacks, there is considerable interest in developing novel algorithms for quantifying MBF with SPECT.

\section{THE EFFECT OF DIABETES ON CORONARY MICROVASCULATURE}

Not surprisingly, the study by Adamu et $\mathrm{al}^{10}$ showed that diabetes was a significant predictor of MACE. Diabetes is known not only to cause microvascular dysfunction, but also increased vascular oxidative stress, which is pro-inflammatory and pro-atherogenic, and may be an important etiology in the development of microangiopathy. In a recent study by Fredrickson et al increased levels of IgG antibodies against malondialdehyde (MDA) modified apoB-100 epitopes P45 and P210 have been demonstrated in diabetic patients suffering from retinopathy and peripheral neuropathy ${ }^{26}$; on the other hand autoantibodies against native apoB-100 epitopes of p45 and p210 are associated with lower prevalence and progression of coronary artery atherosclerosis assessed by coronary calcium imaging. The potential of these auto-antibodies to serve as bio-markers of microangiopathy is evident. The impact of diabetes and insulin resistance on endothelial dysfunction has also been studied before. ${ }^{27,28}$ In patients with diabetes, impairment of endothelium dependent vasodilatation in coronary resistance vessels $(<450 \mu \mathrm{m}$ in diameter) can manifest perfusion abnormalities, even in the absence of angiographic coronary stenosis in the major epicardial vessels. ${ }^{29}$ In a recent study by Soman et al, ${ }^{30} 36$ patients with reversible perfusion defects on MPS and chest discomfort but insignificant CAD $(<50 \%$ stenosis on angiography) were compared with a similar cohort of patients with chest discomfort but normal MPS. The group of patients with reversible MPS defects were found to have significantly less endothelium dependent vasodilatation compared to the control group. On the contrary, there was no difference in endothelium independent vasodilatation with glyceryl tri-nitrate in both the groups. These studies have raised as many questions as they have answered.

\section{CLINICAL IMPLICATIONS}

It is important to consider the clinical implications of the study by Adamu et al; in particular, how will this finding effect patient management, where significant perfusion abnormalities are detected in those with 
nonsignificant angiographic disease? In the absence of an obvious target for revascularization, aggressive riskfactor modification should remain the mainstay of treatment, together with closer clinical follow-up, as we now know that these patients carry a relatively poor prognosis. However, some important questions need to be answered: such as, how often should these patients be followed and what tests should one consider appropriate to monitor disease progression? We believe that a combination of bio-markers, noninvasive modalities assessing endothelial dysfunction and SPECT \pm CT coronary angiography will significantly assist the clinician in managing this interesting cohort of patients. Further clinical trials will be required to clarify these important questions and also provide valuable insight into the pathophysiology of CAD from endothelial dysfunction to overt cardiac events.

\section{References}

1. IMV Medical Information Division. 2005 Nuclear medicine market summary report. Des Plaines, IL: IMV Medical Information Division; 2005.

2. Hachamovitch R, Hayes SW, Friedman JD, Cohen I, Berman DS. Comparison of the short-term survival benefit associated with revascularization compared with medical therapy in patients with no prior coronary artery disease undergoing stress myocardial perfusion single photon emission computed tomography. Circulation 2003;107:2900-7.

3. Shaw LJ, Hachamovitch R, Berman DS, et al. The economic consequences of available diagnostic and prognostic strategies for the evaluation of stable angina patients: An observational assessment of the value of precatheterization ischemia. Economics of Noninvasive Diagnosis (END) Multicenter Study Group. J Am Coll Cardiol 1999;33:661-9.

4. Hachamovitch R, Berman DS, Shaw LJ, et al. Incremental prognostic value of myocardial perfusion single photon emission computed tomography for the prediction of cardiac death: Differential stratification for risk of cardiac death and myocardial infarction. Circulation 1998;97:535-43.

5. Brown KA, Boucher CA, Okada RD, et al. Prognostic value of exercise Tl-201 imaging in patients presenting for evaluation of chest pain. J Am Coll Cardiol 1983;1:994-1001.

6. Ladenheim ML, Pollock BH, Rozanski A, et al. Extent and severity of myocardial hypoperfusion as predictors of prognosis in patients with suspected coronary artery disease. J Am Coll Cardiol 1986;7:464-71.

7. Iskander $\mathrm{S}$, Iskandrian $\mathrm{AE}$. Risk assessment using single-photon emission computed tomographic technetium- $99 \mathrm{~m}$ sestamibi imaging. J Am Coll Cardiol 1998;32:57-62.

8. Berman DS, Hachamovitch $\mathrm{R}$, Kiat $\mathrm{H}$, et al. Incremental value of prognostic testing in patients with known or suspected ischemic heart disease: A basis for optimal utilization of exercise technetium-99m sestamibi myocardial perfusion single-photon emission computed tomography. J Am Coll Cardiol 1995;26:639-47.

9. Soman P, Parsons A, Lahiri N, Lahiri A. The prognostic value of a normal Tc-99m sestamibi SPECT study in suspected coronary artery disease. J Nucl Cardiol 1999;6:252-6.
10. Adamu U, Knollmann D, Almutairi B, et al. Stress/rest myocardial perfusion scintigraphy in patients without significant coronary artery disease. J Nucl Cardiol. doi:10.1007/s12350-009-9133-6.

11. Arnett EN, Isner JM, Redwood DR, Kent KM, Baker WP, et al. Coronary artery narrowing in coronary heart disease: Comparison of cineangiographic and necropsy findings. Ann Intern Med 1979;91(3):350-6.

12. Porter TR, Sears T, Xie F, Michels A, Mata J, et al. Intravascular ultrasound study of angiographically mildly diseased coronary arteries. J Am Coll Cardiol 1993;22(7):1858-65.

13. Nishioka T, Amanullah AM, Luo H, Berglund H, Kim CJ, et al. Clinical validation of intravascular ultrasound imaging for assessment of coronary stenosis severity: Comparison with stress myocardial perfusion imaging. J Am Coll Cardiol 1999;33(7):1870-8.

14. Falk E, Shah PK, Fuster V. Coronary plaque disruption. Circulation 1995;92:657-71.

15. Corti R, Hutter R, Badimon JJ, Fuster V. Evolving concepts in the triad of atherosclerosis, inflammation and thrombosis. J Thromb Thrombol 2004;17:35-44.

16. Anand DV, Lim E, Darko D, et al. Determinants of progression of coronary artery calcification in type 2 diabetes role of glycemic control and inflammatory/vascular calcification markers. J Am Coll Cardiol 2007;50:2218-25.

17. Brown KA, Osbakken M, Boucher CA, et al. Positive exercise thallium-201 test responses in patients with less than 50\% maximal coronary stenosis: Angiographic and clinical predictors. Am J Cardiol 1985;55:54-7.

18. Rozanski A, Gransar H, Wong ND, et al. Clinical outcomes after both coronary calcium scanning and exercise myocardial perfusion scintigraphy. J Am Coll Cardiol 2007;49:1352-61.

19. Pitkanen OP, Raitakari OT, Niinikoski $\mathrm{H}$, et al. Coronary flow reserve is impaired in young men with familial hypercholesterolemia. J Am Coll Cardiol 1996;28:1705-11.

20. Laine H, Raitakari OT, Niinikoski H, et al. Early impairment of coronary flow reserve in young men with borderline hypertension. J Am Coll Cardiol 1998;32:147-53.

21. Tsujimoto G. Impaired coronary microvascular function in diabetics. Ann Nucl Med 2000;14:165-72.

22. Storto G, Sorrentino AR, Pellegrino T, et al. Assessment of coronary flow reserve by sestamibi imaging in patients with typical chest pain and normal coronary arteries. Eur J Nucl Med Mol Imaging 2007;34:1156-61.

23. Abe Y, Kondo M, Kubota T, et al. Non-invasive assessment of coronary microvascular dysfunction using $\mathrm{Tc} 99 \mathrm{~m}$ Tetrofosmin SPECT in patients with acute myocardial infarction. J Nucl Cardiol 2004;11:562-9.

24. Maddahi J, Abdulla A, Garcia EV, et al. Non-invasive identification of left main and triple vessel coronary artery disease: Improved accuracy using quantitative analysis of regional myocardial stress distribution and washout of Thallium-201. J Am Coll Cardiol 1986;7:53-60.

25. Okada RD, Glover D, Gaffney T, et al. Myocardial kinetics of Technetium 99m-hexakis-2-methoxy-methylpropyl-isonitrile. Circulation 1988;77:480-91.

26. Fredrikson GN, Anand DV, Hopkins D, et al. Associations between autoantibodies against apolipoprotein B-100 peptides and vascular complications in patients with type 2 diabetes. Diabetologia 2009;52:1426-33.

27. Angeja BG, de Lemos J, Murphy SA, et al. TIMI study group (2002) Thrombolysis in myocardial infarction; impact of diabetes mellitus on epicardial and micro-vascular flow after fibrinolytic therapy. Am Heart J 2002;144:649-56.

28. Kjaer A, Meyer C, Nielsen FS, et al. Dipyridamole, cold pressor test and demonstration of endothelial dysfunction: A 
PET study of myocardial perfusion in diabetes. J Nucl Med 2003;44:19-23.

29. Marcus ML, Chilian WM, Kanatsuka H, et al. Understanding the coronary circulation through studies at the micro-vascular level. Circulation 1990;82:1-7.
30. Soman P, Dave DM, Udelson JE, et al. Vascular endothelial dysfunction is associated with reversible myocardial perfusion defects in the absence of obstructive coronary artery disease. J Nucl Cardiol 2006;13:756-60. 